\title{
ANÁLISE DE EFEITO DA POLÍTICA PÚBLICA NO DESENVOLVIMENTO DOS APLS DE MINAS GERAIS
}

\section{EFFECT ANALYSIS OF PUBLIC POLICY ON THE DEVELOPMENT OF LPAS IN MINAS GERAIS}

\author{
Cecilia Alves da Silva Antero \\ UFV - Universidade Federal de Viçosa - MG \\ cecilia.antero@yahoo.com.br \\ Magnus Luiz Emmendoerfer \\ UFV - Universidade Federal de Viçosa - MG \\ magnus@ufv.br \\ Valdir Roque Dallabrida \\ Universidade do Contestado - UNC - Santa Catarina \\ valdirdallabrida@gmail.com
}

Submissão: 16/12/2019

Aprovação: 29/10/2020

Acknowledgements: National Council for the Improvement of Higher Education - Brazil (CAPES) for financial support. Research Support Foundation of Minas Gerais (FAPEMIG) for financial support. National Council for Research and Development $(\mathrm{CNPq})$ for support.

Agradecimentos: Coordenação de Aperfeiçoamento de Pessoal de Nível Superior - Brasil (CAPES) pelo apoio financeiro. Fundação de Amparo à Pesquisa do Estado de Minas Gerais (FAPEMIG) pelo apoio financeiro. Conselho Nacional de Desenvolvimento Científico e Tecnológico $(\mathrm{CNPq})$ pelo apoio para a realização da pesquisa.

\section{RESUMO}

O objetivo foi analisar o efeito da política pública no desenvolvimento dos APLs de Minas Gerais, Brasil. Foram utilizados dados dos 853 municípios do estado, referentes ao período de 2005 a 2016, extraído das bases FIRJAN e IMRS FJP. Para o tratamento e análise dos dados utilizou-se o software Stata ${ }^{\circledR}$. Para capturar esse efeito, foi empregado um modelo de efeitos fixos com dummy. Verificou-se, nos municípios com APLs beneficiados pela referida política, um efeito positivo e significativo, indicando incremento de $9,35 \%$ nos indicadores de desenvolvimento. Tais resultados oferecem uma sinalização positiva para a continuidade da política analisada, ou de ações semelhantes. Os APLs são reconhecidos como vetores de desenvolvimento muito em função de sua capacidade de potencializar a geração de emprego e renda. Assim, entende-se que a manutenção de ações para fortalecer as economias locais ao apoiar seu desenvolvimento tende a implicar positivamente no contexto onde eles estão inseridos.

Palavras-chave: Administração Pública Municipal. Desenvolvimento. Gastos Públicos. Investimentos. Brasil. 
ABSTRACT

The objective was to analyze the effect of public policy on the development of Minas Gerais LPAs in Brazil. We used data from 853 municipalities of the state, for 2005 to 2016, extracted from the bases FIRJAN and IMRS FJP. For data treatment and analysis, the Stata ${ }^{\circledR}$ software was used. To capture this effect, a dummy fixed effects model was employed. In the municipalities belonging to LPAs benefiting from this policy, there was a positive and significant effect, indicating a 9.35\% increase in development indicators. These results provide a positive signal for the continuity of the policy under analysis, or similar actions. LPAs are recognized as vectors of development largely because of their ability to enhance employment and income generation. Thus, it is understood that maintaining actions to strengthen local economies by supporting their development tends to positively imply the context in which they are inserted.

Keywords: Municipality Public Administration. Development. Public spending. Investments. Brazil.

\section{INTRODUÇÃO}

Os Arranjos Produtivos Locais (APLs) são reconhecidos como vetores de desenvolvimento (AZEVEDO FILHO; RIBEIRO, 2011; SANTOS; DINIZ; BARBOSA, 2004; BAPTISTA; ALVAREZ, 2007; BARROSO; SOARES, 2009; LASTRES; CASSIOLATO, 2003; ZAMBRANA; TEIXEIRA, 2013).

Em função da mudança do foco de atuação do poder público das grandes empresas para as economias locais (TEIXEIRA, 2008; CASTRO; GONÇALVES, 2014), os APLs foram identificados como um espaço para implementação de ações com objetivo de fomentar o desenvolvimento. Como exemplo cita-se as políticas públicas de apoio ao desenvolvimento dos APLs, no nível federal (BRASIL, 2004) e estadual (MINAS GERAIS, 2006).

Entende-se que, no APL, o poder público tem a função de atenuar os problemas de baixo dinamismo das economias regionais por meio de atividades voltadas para a melhoria das condições de crescimento econômico e desenvolvimento tecnológico, com objetivo de tornar as empresas competitivas, favorecendo assim a geração de emprego e renda (BAPTISTA; ALVAREZ, 2007; CAMPOS; TRINTIN; VIDIGAL, 2009).

Com base nas políticas federal e estadual de apoio aos APLs (BRASIL, 2004; MINAS GERAIS, 2006), sabe-se que as ações do poder público devem concentrar-se na realização de investimentos nas atividades desenvolvidas, na infraestrutura/logística e na qualificação.

Porém, apesar dos trabalhos debaterem sobre a importância da atuação comprometida por parte dos representantes do poder público (CASTRO; GONÇALVES, 2014; TEIXEIRA, 2008) e do destaque dado às situações problemáticas em função da ausência dessa atuação (ALMEIDA; ALBINO 2011; OLIVEIRA; SANTANA, 2012; PEREIRA; GUIMARÃES; GOMES, 2011), poucos estudos fazem referência à influência das ações do poder público, no desenvolvimento dos APLs, por meio de investimentos de diversas ordens.

Neste contexto, definiu-se como objetivo analisar o efeito da política pública no desenvolvimento dos APLs de Minas Gerais. Para tanto, optou-se pela análise de regressão com dados em painel para identificar tal efeito. Parte-se do pressuposto de que as ações do poder público, no que tange à realização de investimentos, têm efeitos positivos nos indicadores de desenvolvimento.

Ao compreender em que medida os investimentos realizados influenciam o desenvolvimento será possível mitigar algumas limitações quanto aos entendimentos que 
permeiam esses temas e identificar as ações mais adequadas a um contexto, de efeito positivo ou não, quanto aos investimentos realizados.

Esse trabalho é organizado em duas seções, além desta introdução, Parte 1, compreendendo o referencial teórico, onde se discute sobre políticas públicas e desenvolvimento local e, Parte 2, abrangendo procedimentos metodológicos e análise dos resultados, onde se explica como o estudo foi realizado e os resultados obtidos.

\section{POLÍTICAS PÚBLICAS E DESENVOLVIMENTO}

Nesta seção serão apresentados entendimentos sobre desenvolvimento local e políticas públicas de apoio ao desenvolvimento dos APLs como alternativa para facilitar o alcance do objetivo proposto.

\section{Desenvolvimento Local ${ }^{1}$}

Nos anos de 1990 a promoção do desenvolvimento tornou-se bem mais complexa que a produção interna e a oferta de bens e serviços padronizados para o mercado doméstico. Observou-se a necessidade de considerar a importância da diferenciação, da cooperação, da participação e da obtenção de qualidade de vida. Neste contexto, as ações em prol do desenvolvimento deixaram de ser pensadas de forma abrangente para considerar a localidades (COSTA; CUNHA, 2002).

O desenvolvimento local compreende a valorização da economia e dinâmica local, por meio da utilização de recursos endógenos, com o objetivo de promover o crescimento dessa economia, a criação de emprego e a melhoria da qualidade de vida (COMITÉ, 1995; MARTINS, 2002; MACEDO; ARAÚJO; CAMPOS, 2014).

Martins (2002) afirma que para compreender o desenvolvimento local é preciso definir o contexto, determinado pela escala das inter-relações que ocorrem cotidianamente entre atores sobre um determinado espaço geográfico, a partir das quais se constrói uma identidade. Segundo o autor, é preciso observar a dinâmica e historicidade das inter-relações entre atores, as possibilidades sociais e econômicas onde ocorrem tais inter-relações, bem como as atividades desempenhadas tendo em vista a manutenção de sua sobrevivência por meio do uso dos recursos locais.

Entende-se que o desenvolvimento local, fomentado pelos representantes do poder público local, ocorre na realização de atividades produtivas por meio da utilização de recursos, considerando as características do local. É fruto dos diversos esforços e compromissos dos atores locais (ALBUQUERQUE, 1998).

As ações em prol do desenvolvimento local devem ter como objetivo: (i) estimular e detectar iniciativas produtivas; (ii) facilitar às empresas locais o acesso negociado aos serviços avançados de apoio à produção; (iii) estabelecer mecanismos para o financiamento de novas empresas e uma oferta de crédito compatível com as características da PME e da microempresa e (iv) incentivar a cooperação interempresarial no território (ALBUQUERQUE, 1998).

Apesar das ações serem desenvolvidas no local, o poder público, nos âmbitos estadual e federal, deve compartilhar as responsabilidades quanto à sua execução e aos resultados obtidos (ALBUQUERQUE, 1998). Macedo, Araújo e Campos (2014) reconhecem que o envolvimento do poder público tende a fortalecer a estrutura e proporcionar maior competitividade aos APLs. Jacometti et al. (2014) destacam que bons resultados só serão alcançados se os representantes do poder público tiverem preparação e flexibilidade na realização de ações em prol do desenvolvimento.

Martins (2002) enfatiza que uma característica do desenvolvimento local é a inserção 
dos atores locais, assegurando-lhes o papel de realizadores e não apenas beneficiários. Em adição, Albuquerque (1998) destaca a importância do envolvimento dos atores locais e da coordenação das ações em prol do desenvolvimento local. Segundo o autor, cabe ao poder público a responsabilidade pela coordenação, sendo que as iniciativas locais podem ser na área do desenvolvimento tecnológico, na formação de recursos humanos específicos ou iniciativas de financiamento focadas em ações de desenvolvimento local.

Neste trabalho, consideram-se as iniciativas relacionadas ao financiamento, destacando o papel dos representantes do poder público que têm responsabilidade de permitir aos representantes das micro e pequenas empresas acesso às linhas de financiamentos ajustadas às circunstâncias e características locais. Tal entendimento é materializado nos APLs, uma vez que os atores locais desempenham atividades conforme vocação local para gerar desenvolvimento.

As iniciativas locais de financiamento de ações ou iniciativas de desenvolvimento, ou seja, as ações do poder público em Minas Gerais em prol do desenvolvimento local, estão concentradas na concessão de investimentos para fomentar a dinamização dos APLs.

Sobre a relação dos gastos públicos e desenvolvimento, de forma geral, há um entendimento entre os autores de que tais investimentos podem influenciar positivamente nos indicadores de desenvolvimento, por meio do aumento da produtividade do setor privado (ANDRADE; TEIXEIRA; FORTUNATO, 2014; BOGONI; HEIN; BEUREN, 2011; DEGENHART et al., 2016; SOUSA; PAULO, 2016). Andrade, Teixeira e Fortunato (2014) constataram que podem ser promovidos efeitos positivos no desenvolvimento municipal por meio dos gastos públicos nas áreas de saúde e saneamento. Degenhart et al. (2016) afirmam que os efeitos gerados pelos investimentos públicos resultam na geração de emprego e renda e melhor aplicação dos recursos públicos. Já Bogoni, Hein e Beuren, 2011 (2011) afirmam que os gastos dos governos locais têm um importante papel no crescimento econômico, enquanto Sousa e Paulo (2016) destacam que os resultados indicam que, tomados em conjunto, os gastos agregados dos estados brasileiros apresentam-se positivamente relacionados ao crescimento econômico.

\section{Arranjos Produtivos Locais}

Um APL consiste numa "[...] aglomeração territorial de atores econômicos, políticos e sociais com foco em um conjunto específico de atividades econômicas que apresentam vínculos mesmo que incipientes" (LASTRES; CASSIOLATO, 2003, p. 3). Apesar das diversas nomenclaturas identificadas (HASENCLEVER; ZISSIMOS, 2006), no Brasil prevalece a utilização do termo APL (ALMEIDA; ALBINO, 2011; AZEVEDO FILHO; RIBEIRO, 2011; SANTOS; CÂNDIDO, 2013), uma vez que tal fenômeno manifesta-se de forma recorrente.

Os elementos importantes para a definição APL são: i) concentração de atores (representando a reunião de diversas empresas que desenvolvem atividades complementares e entidades de apoio); ii) localização num mesmo território (enfatizando a necessidade de pertencerem a um mesmo espaço) e iii) existência de vínculos de cooperação, interação, articulação, aprendizado (BAPTISTA; ALVAREZ, 2007; VALE, 2007; LASTRES; CASSIOLATO, 2003; SEBRAE, 2003). A caracterização de um APL, nesse projeto, é baseada na identificação de quatro fatores fundamentais: a concentração setorial e territorial, a existência de entidades, o relacionamento entre atores e a obtenção de benefícios (Quadro 01).

Quadro 1: Fatores para identificação de um APL

\begin{tabular}{|l|l|}
\hline \multicolumn{1}{|c|}{ Fator } & \multicolumn{1}{c|}{ Descrição } \\
\hline $\begin{array}{l}\text { Concentração } \\
\text { setorial e territorial }\end{array}$ & $\begin{array}{l}\text { A concentração territorial implica na presença de empresas em um mesmo espaço, uma } \\
\text { vez que o território consiste numa rede de relações que se projetam num determinado } \\
\text { espaço constituído por relações políticas, socioeconômicas e culturais. Já a }\end{array}$ \\
\hline
\end{tabular}




\begin{tabular}{|l|l|}
\hline & $\begin{array}{l}\text { concentração setorial permite identificar o tipo de atividade desempenhada pelas } \\
\text { empresas, em que são destacadas atividades diferentes, porém correlatas e } \\
\text { complementares (LASTRES; CASSIOLATO, 2003). }\end{array}$ \\
\hline $\begin{array}{l}\text { Existência de } \\
\text { entidades }\end{array}$ & $\begin{array}{l}\text { Organizações públicas, privadas e não governamentais cujo papel é fomentar o } \\
\text { desenvolvimento do APL (LASTRES; CASSIOLATO, 2003; SEBRAE, 2003) e } \\
\text { proporcionar mais competitividade por meio da intensificação de relação com os atores } \\
\text { (BAPTISTA; ALVAREZ, 2007). }\end{array}$ \\
\hline $\begin{array}{l}\text { Relacionamento } \\
\text { entre atores }\end{array}$ & $\begin{array}{l}\text { Vínculos de cooperação, interação e articulação condicionados por uma série de } \\
\text { características inerentes ao APL e pela participação e interação de empresas, } \\
\text { organizações de apoio, clientes, fornecedores e concorrentes (LASTRES; } \\
\text { CASSIOLATO, 2003). }\end{array}$ \\
\hline $\begin{array}{l}\text { Obtenção de } \\
\text { benefícios }\end{array}$ & $\begin{array}{l}\text { Os benefícios adquiridos de maneira passiva são um subproduto incidental e não } \\
\text { intencional da atividade (MARSHALL, 1982) e ocorrem pela concentração setorial em } \\
\text { determinado território. Os benefícios adquiridos de maneira ativa representam a } \\
\text { vantagem competitiva derivada da ação conjunta (SCHMITZ, 1997) e requerem ações } \\
\text { propositais, exigindo esforço por parte dos membros do APL para fomentar seu } \\
\text { desenvolvimento. }\end{array}$ \\
\hline
\end{tabular}

Fonte: Elaboração própria

Os APLs têm importante papel no desenvolvimento das empresas devido à possibilidade de obtenção de benefícios oriundos da concentração setorial e territorial e das formas de relacionamento entre atores, à exemplo da especialização, maior dinamismo nos mercados, geração de conhecimento, vínculos de interação, cooperação e aprendizagem (CASSIOLATO; LASTRES, 2001; AQUINO; BRESCIANI, 2005; PASSOS et al., 2005; VALE, 2007; ALMEIDA; ALBINO, 2011; DIAS, 2013; SOUZA et al. 2015). Segundo Maria, Faria e Amorim (2008) e Sugahara e Vergueiro (2012), o APL é um ambiente favorável à socialização e obtenção de conhecimento tácito.

Além desses benefícios, obtidos de forma passiva devido à presença das empresas no APL, a ação proposital das entidades pode gerar promoção de capacitação e formação, criação de consórcios de exportação e de centros tecnológicos e aumento da capacidade de inovação local, dentre outros (SUZIGAN et al., 2003; CASSIOLATO; LASTRES, 2001; KREUZ et al., 2005; CAMPOS; TRINTIN; VIDIGAL, 2009).

Apesar dos benefícios proporcionados, em um APL podem ser identificados desafios relativos à condução e organização de ações conjuntas o que requer a identificação de alternativas para minimizá-los e facilitar a atuação dos atores (KACHBA; HATAKEYAMA, 2013; FUINI, 2014; TAHIM; ARAÚJO JUNIOR, 2014).

\section{Políticas Públicas de apoio ao desenvolvimento dos APLs}

As ações integradas de políticas públicas para APLs consistem em uma das estratégias do poder público federal para fomentar o desenvolvimento. Para mudar a lógica da atuação individualizada de órgãos governamentais e não governamentais, seus representantes, em geral, se empenham em criar certa conscientização quanto à importância de uma atuação coletiva, coordenada, articulada e complementar (BRASIL, 2004; TEIXEIRA, 2008; CASTRO; GONÇALVES, 2014).

Um resultado dessa iniciativa foi a criação, em 2003, do Grupo de Trabalho Permanente para APL (GTP APL) que tem como questões centrais os elementos: cooperação, aprendizado coletivo, conhecimento tácito e capacidade inovativa das empresas e instituições locais, as quais devem atuar de forma interdependente para aumentar competitividade e fortalecer a governança (BRASIL, 2004). O GTP APL foi formalizado pela Portaria Interministerial $\mathrm{n}^{\mathrm{o}} 200$ de 02 de agosto de 2004, do Ministério do Desenvolvimento, Indústria e Comércio Exterior (MDIC), com o objetivo elaborar e propor diretrizes gerais para a 
atuação coordenada do poder público federal no apoio ao desenvolvimento dos APLs em todo o território nacional.

A atuação de representantes de diversos ministérios e outras instituições em um movimento a favor da abordagem de APL resultou no reconhecimento da importância dos APLs por meio da inclusão do tema como política de governo no Plano Plurianual de 20042007 e nos documentos que tratam das políticas nacionais de desenvolvimento (BRASIL, 2004).

A opção do poder público federal de apoiar os APLs se deve ao reconhecimento de que as políticas de fomento ao desenvolvimento de grupos de empresas são mais efetivas do que quando direcionadas às empresas individualmente, uma vez que a competitividade das empresas que atuam coletivamente é resultado dos ganhos provenientes da maior cooperação entre firmas (capacidades produtivas e inovativas e incremento do capital social). Outro fator que justifica a atenção destinada aos APLs é a dimensão local, pois as atividades desempenhadas pelas empresas refletem vocações locais e muitas vezes utilizam recursos também locais (BRASIL, 2004; TEIXEIRA, 2008).

No estado de Minais Gerais, o poder público estadual instituiu a política Estadual de apoio aos APLs, em 01 de agosto de 2006, por meio da Lei 16.296, a fim de promover o fortalecimento das economias regionais por meio da integração e da complementaridade das cadeias produtivas locais e geração de processos permanentes de cooperação, difusão e inovação (MINAS GERAIS, 2006, 2008). As ações integradas para os APLs de Minas Gerais estão concentradas no Programa de Apoio à Competitividade dos APLs de Minas Gerais (PAC APL MG) das Superintendências de apoio à competitividade e ao empreendedorismo e de apoio aos APLs (MINAS GERAIS, 2015).

Esse programa objetiva aumentar a produtividade e a competitividade das empresas envolvidas nos APLs participantes. Os objetivos específicos do programa são: (i) fomentar a articulação das entidades federais, estaduais e locais promotoras de APLs; (ii) elaborar os planos de melhoria da competitividade para os APLs selecionados; (iii) implementar as atividades propostas nos PACs e (iv) implantar um sistema de monitoramento, avaliação e identificação das lições aprendidas, que permita fazer uma análise e difusão dos efeitos e impactos do programa (MINAS GERAIS, 2015).

A efetivação do PAC APL MG ocorre por meio da participarão dos APL beneficiados nas atividades previstas no Plano de Melhoria da Competitividade (PMC), que contempla o diagnóstico da situação atual do APL, estratégias de atuação e atividades a serem implementadas conforme linhas de atuação. Tais linhas de atuação, que compreendem ações financiadas pelo PAC APL MG, para que os APLs conquistem novos mercados e adotem práticas sustentáveis de produção, são capacitação e assessoria empresarial, comercialização, prospecção de mercado e exportações, gestão e organização do APL, logística, meio-ambiente e desenvolvimento social e tecnologia e inovação (MINAS GERAIS, 2015).

Foram identificados 34 APLs, com base nos critérios de relevância da base empresarial, participação do APL na economia da região, nível de cooperação e colaboração entre as empresas do APL, perspectivas para o setor e empresas da região, perspectivas de geração de empregos e empregos de qualidade (MINAS GERAIS, 2015).

Esses APLs estão distribuídos em 174 cidades e suas atividades concentram-se nos setores de bebidas artesanais (cachaça de alambique), biotecnologia, calçados, calçados e bolsas, confecções, eletroeletrônico, floricultura, fogos de artifício, fruticultura, fundição, gemas e artefatos em pedras, gemas e joias, móveis, pedra sabão, quartzito (BOTELHO, 2009). Entretanto, em função da limitação de recursos financeiros, somente sete APLs foram beneficiados pelo programa: APL Fruticultura do Jaíba, APL Fundição de Cláudio, APL Biotecnologia da região metropolitana de Belo Horizonte (RMBH), APL Calçados e Bolsas da RMBH, APL Eletroeletrônicos de Santa Rita do Sapucaí, APL Móveis de Ubá e APL 
Calçados Nova Serrana (MINAS GERAIS, 2015), que consistem no objeto de estudo deste trabalho.

\section{PROCEDIMENTOS METODOLÓGICOS E ANÁLISE DOS DADOS}

Nessa seção são apresentadas as escolhas feitas quanto aos procedimentos utilizados para sua construção, bem como os resultados obtidos.

Foram investigadas unidades de análise múltiplas, dada a necessidade de observar informações sobre os 853 municípios do Estado de Minas Gerais, considerando o período de 2006 a 2016. Foram analisadas as informações de todos os municípios, dando ênfase aos que fazem parte dos APLs beneficiados pela Política Pública de Apoio aos APLS do estado de Minas Gerais.

A opção por analisar o referido período é fundamentada na data de início da política pública no estado, em 2006, na existência de informações sobre a execução da política, até 2016, e na existência de dados relativos às variáveis consideradas para análise, até 2016. Em relação à unidade de análise, escolheu-se Minas Gerais em função da existência da política pública de apoio aos APLs, pela participação desse estado na economia do país e pela alta concentração de pequenas e médias empresas que atuam em diversos setores da economia (GARONE et al., 2015).

As variáveis do modelo são descritas a seguir no Quadro 2.

Quadro 2: Variáveis utilizadas no estudo

\begin{tabular}{|c|c|c|c|c|c|}
\hline \multicolumn{2}{|c|}{ Variável } & Descrição & Fonte & Efeito & Base Teórica \\
\hline Dependente & IFDM & Proxy de desenvolvimento & FIRJAN & & \\
\hline
\end{tabular}

\begin{tabular}{|l|l|l|l|l|l|}
\hline Independente & INVEST & Dummy para o investimento & & + & $\begin{array}{l}\text { Jacometti } \text { et al. } \text { (2016) e } \\
\text { Macedo, Araújo e Campos } \\
(2014)\end{array}$ \\
\hline
\end{tabular}

\begin{tabular}{|c|c|c|c|c|c|}
\hline & GPEDU & Gastos com educação & \multirow{10}{*}{ IMRS FJP } & + & Medeiros et al. (2018) \\
\hline & & Gastos com saúde & & & Andrade, Teixeira e \\
\hline & GPSAU & & & + & $\begin{array}{l}\text { Fortunato }(2014) \mathrm{e} \\
\text { Degenhart et al }(2016)\end{array}$ \\
\hline & GPREN & Gastos com renda & & & Kühn e Tonetto (2017) \\
\hline & GPINF & Gastos com infraestrutura & & + & Gonçalves et al. (2017) \\
\hline & GPTRA & Gastos com trabalho & & & Degenhart et al. (2016) \\
\hline \multirow{4}{*}{ Controle } & GPSEG & Gastos com segurança & & + & Schull et al. (2014) \\
\hline & GPHAB & $\begin{array}{l}\text { pública } \\
\text { Gastos com habitação }\end{array}$ & & $\begin{array}{l}+ \\
+\end{array}$ & Neduziak e Correia (2017) \\
\hline & GPASC & $\begin{array}{l}\text { Gastos com assistência social } \\
\text { e cidadania }\end{array}$ & & + & Degenhart et al. (2016) \\
\hline & GPDEC & $\begin{array}{l}\text { Gastos com desenvolvimento } \\
\text { econômico }\end{array}$ & & + & Medeiros et al. (2018) \\
\hline
\end{tabular}

Fonte: Elaboração própria

Nota: A variável INVEST possibilitará captar o efeito dos investimentos realizados nos APLs beneficiados pela Política Pública de Apoio aos APLS do estado de Minas Gerais. Essa variável assume valor de 0 para não investimento e 1 para investimento, com controle para ano e munícipio. 
Para o tratamento e a análise dos dados contou-se com o software Stata ${ }^{\circledR}$. Adotou-se a análise de painel de dados (HILL; GRIFFITHS; LIM, 2011) num modelo estatístico de regressão múltipla linear. Esse tipo de análise possibilita analisar a relação entre variáveis, inferir eventuais diferenças entre os municípios mineiros, considerando as variáveis observadas e perceber ao longo do período as variações provocadas pelos investimentos realizados nos APLs beneficiados. Para tanto, utilizou-se um modelo de efeitos fixos com variável dummy:

$\mathrm{IFDM}_{i t}=\alpha_{i}+\beta 1 \mathrm{GPEDU}_{i t}+\beta 2 \mathrm{GPSAU}_{i t}+\beta 3 \mathrm{GPREN}_{i t}+\beta 4 \mathrm{GPINF}_{i t}+\beta 5 \mathrm{GPTRA}_{i t}+\beta 6 \mathrm{GPSEG}_{i t}+$ $\beta 7 \mathrm{GPHAB}_{\text {it }}+\beta$ GGPASC $_{\text {it }}+\beta 10 \mathrm{GPDEC}_{\text {it }}+\operatorname{dINVEST}_{\mathrm{it}}+\mathrm{e}_{\mathrm{it}}$

Em que os $\beta$ capturam o efeito dos gastos públicos per capta em educação, saúde, renda, infraestrutura, trabalho, segurança, habitação, assistência social e cidadania e desenvolvimento econômico; o $d$ o efeito dos investimentos realizados nos APLs beneficiados pela Política Pública de Apoio aos APLS do estado de Minas Gerais ${ }^{2} ; e_{\text {it }}$ representa a aleatoriedade do modelo. Espera-se um efeito positivo das variáveis de controle e independente, uma vez que investimentos tendem a promover incrementos no desenvolvimento.

\section{ANÁLISE DOS RESULTADOS}

Quanto à influência dos investimentos realizados, a seguir na Tabela 1, apresenta-se um sumário dos resultados obtidos a partir da estimação do Modelo 1. As estimativas foram alcançadas por meio do modelo de efeitos fixos com variável dummy, considerado estatisticamente significativo $(\mathrm{F}(10,9369)=216.37 \mathrm{e}$ Prob $>\mathrm{F}=0.0000)$. Para confirmar a adequação do modelo utilizado, realizou o Teste de Chow em que se confirmou a predominância do modelo em relação do modelo Pool (estatísticas: F $(10,8517)=773.13$ e Prob $>\mathrm{F}=0.0000)$ e Teste de Hausman, que também demonstrou que o modelo é mais adequado que o Modelo de Efeitos Aleatórios (estatísticas: 735.98 Chi-sq (10) P-value = 0.0000).

Tabela 1: Influência dos investimentos no período de 2006 a 2016.

\begin{tabular}{|c|c|c|c|}
\hline Vari & & Coeficiente & $P>|t|$ \\
\hline endente ${ }^{\text {Indep }}$ & NVEST & .0935036 & 0.000 \\
\hline
\end{tabular}

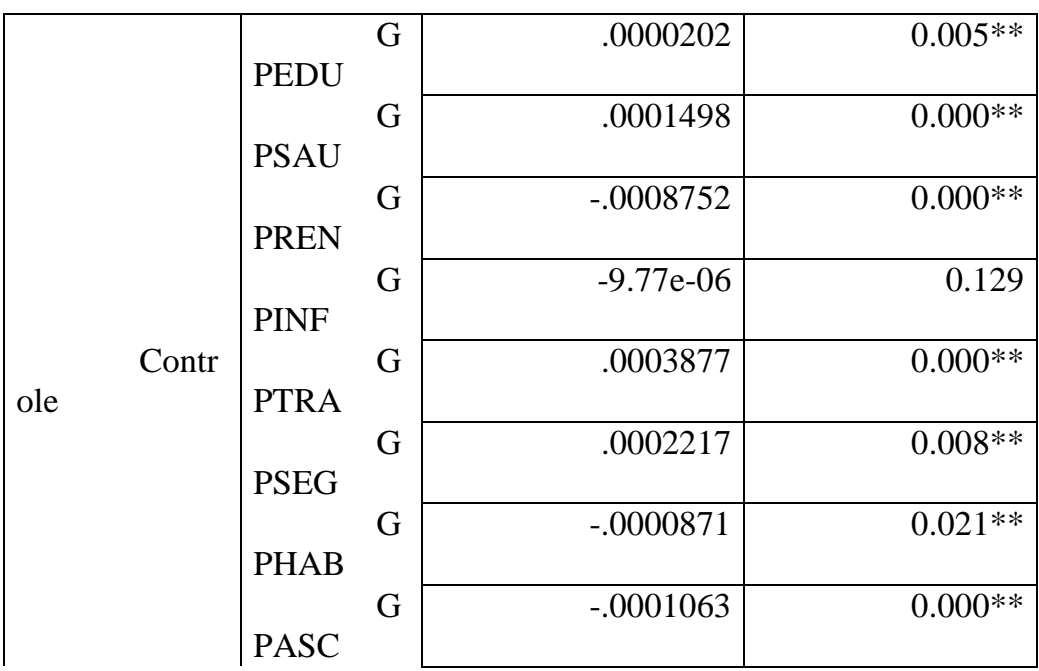




\begin{tabular}{|l|r|r|r|}
\cline { 3 - 4 } & PDEC & .0002206 & $0.000^{* *}$ \\
\hline _cons & .5904809 & $0.000^{* *}$ \\
\hline
\end{tabular}

Fonte: Elaboração própria.

Nota: $\mathrm{O} * *$ denota uma significância de $5 \%$

O Modelo 1 foi empregado para dar ênfase aos indicadores de desenvolvimento, considerando os municípios que fazem parte dos APLs beneficiados pela Política Pública de Apoio aos APLS do estado de Minas Gerais.

Sobre as variáveis de controle, observou-se somente que o GPINF não apresentou efeito significativo, sendo que GPASC, GPHAB e GPREN apresentaram efeito significativo e negativo e GPDEC, GPEDU, GPTRA, GPSAU e GPSEG apresentaram efeito significativo e positivo. Esse resultado confirma a adequação das variáveis para controlar os efeitos do investimento realizado por meio da política pública em análise.

Os achados da pesquisa corroboram com os estudos de Andrade, Teixeira e Fortunato (2014), Degenhart et al. (2016), Medeiros et al. (2018) e Schull et al. (2014). Entretanto, não confirmam os achados de Degenhart et al. (2016), Kühn e Tonetto (2017) e Neduziak e Correia (2017), para as variáveis GPASC, GPHAB, GPINF e GPREN.

Em relação ao GPASC, acredita-se que o resultado aqui identificado se diferencia do obtido por Degenhart et al. (2016) em função das escolhas realizadas na pesquisa, pois o referido autor optou por analisar os dez maiores e os dez menores municípios da região sudeste e utilizar o PIB como proxy para o desenvolvimento.

No que se refere ao comportamento da variável GPREN, Kühn e Tonetto (2017) analisaram os 497 municípios do Rio Grande do Sul e utilizaram o Índice de Desenvolvimento Humano Municipal (IDHM) como proxy para o desenvolvimento.

Parte-se do mesmo entendimento para justificar a não confirmação dos achados de Neduziak e Correia (2017) sobre GPHAB. Nesse estudo, o autor optou por analisar os estados brasileiros, utilizando o PIB como proxy para analisar o efeito dos gastos públicos no desenvolvimento.

Ressalta-se que esses estudos foram utilizados como referência por oferecerem, a partir dos resultados das pesquisas, indicativos da relação entre os gastos públicos, em áreas específicas, como o desenvolvimento. Isso confirma a adequação das variáveis utilizadas nesse estudo para controlar os efeitos do investimento realizado por meio da política pública em análise. Dito de outra forma, essas variáveis foram inseridas no modelo para facilitar a captação do efeito da política pública no desenvolvimento dos municípios analisados.

Quanto aos investimentos realizados nos APLs, pela Política Pública de Apoio aos APLS do estado de Minas Gerais, considerou-se a condição de beneficiamento para analisar como estes investimentos influenciam o indicador de desenvolvimento.

Percebeu-se que os investimentos realizados proporcionaram um efeito positivo de 9,35\% no indicador de desenvolvimento dos municípios participantes de APLs e beneficiados pela Política Pública de Apoio aos APLS do estado de Minas Gerais, considerando o período de 2006 a 2016.

Isso implica dizer que a presença do investimento influencia o desenvolvimento. Dito de outra forma, os municípios pertencentes a APLs beneficiados pela referida política pública têm um melhor desempenho, no nível de desenvolvimento em relação aos que não são pertencentes a APLs beneficiados.

Esses achados corroboram o entendimento de Macedo, Araújo e Campos (2014) sobre a influência positiva de políticas públicas no desenvolvimento dos APLs. Esses autores investigaram o APL de Plásticos no ABC e apresentaram evidências a partir da interpretação de informações sobre o desempenho do referido APL em sua fase inicial.

Da mesma forma, Jacometti et al., 2016 defenderam que a realização de investimentos 
tende a promover o desenvolvimento. Esses autores, em seu estudo no APL de móveis de Arapongas (PR), destacaram a influência da política pública federal, com base na percepção dos atores do APL, apesar de não terem indicativos do efeito positivo de políticas locais.

É importante destacar que esses e outros estudos, que partem de resultados de pesquisas qualitativas, são motivadores à realização de pesquisas para sistematizar a relação investimento, por meio de políticas públicas, e desenvolvimento no contexto dos APLs. Nesses estudos enfatiza-se que a existência de políticas públicas tende a promover melhores resultados, e que isso normalmente implica positivamente no desenvolvimento dos APLs.

Além dos estudos supramencionados, há o trabalho de Antero, Rodrigues, Emmendoerfer e Dallabrida (2020) que parte desse mesmo entendimento, mas diferencia-se dos demais por suas escolhas metodológicas que possibilitaram a sistematização da análise do impacto de investimentos no desenvolvimento dos APLs. O trabalho confirma a hipótese de que os municípios pertencentes a APLs, quando beneficiados por investimentos apresentam melhores resultados que os municípios não pertencentes a APLs.

Especificamente, quando se aborda o investimento, entende-se que há uma propensão em aumentar a geração de emprego e renda, principalmente em APLs. Os estudos realizados por Baptista e Alvarez (2007) e Campos, Trintin e Vidigal (2009) enfatizam a importância da ação da administração pública, no que tange a realização de ações para fomentar o desenvolvimento dos APLs tendo em vista a elevação do número de emprego e renda.

\section{CONSIDERAÇÕES FINAIS}

O objetivo deste trabalho foi analisar o efeito da política pública na evolução dos APLs de Minas Gerais. Partiu-se do pressuposto que as ações do poder público, no que tange à realização de investimentos, têm efeitos positivos na melhoria dos indicadores de desenvolvimento.

Considerando os municípios beneficiados pela Política Pública de Apoio aos APLs do estado de Minas Gerais observou-se um efeito positivo e significativo, indicando que o apoio destinado aos APLs proporciona elevação no nível de desenvolvimento.

Destaca-se também que, pela natureza do estudo realizado, os resultados encontrados diferenciam-se dos estudos realizados anteriormente em função do período analisado, das variáveis utilizadas para análise e do modelo empregado. Dessa forma, este estudo teve uma contribuição, no sentido de realizar uma investigação embasada em formas alternativas para compreender o desenvolvimento no contexto municipal. Especificamente, quando se considera a influência dos investimentos nos indicadores de desenvolvimento, este estudo demonstra de forma sistematizada o efeito da Política Pública de Apoio aos APLS do estado de Minas Gerais ao indicar que os municípios beneficiados se diferenciam quanto ao nível de desenvolvimento.

Quanto aos procedimentos adotados, entende-se que foram adequados, pois as variáveis utilizadas serviram de base para estudos anteriores, os testes confirmam a capacidade de explicação do modelo empregado, justificativas necessárias para a escolha de modelos adequados.

Apesar das limitações relativas à impossibilidade de considerar um período maior em função da indisponibilidade de dados e à possibilidade de existência de outras variáveis para explicar o efeito dos gastos públicos nos indicadores de desenvolvimento, em especial na dimensão socioeconômica, considera-se que o estudo avança na compreensão desta temática. Assim, fica demonstrado que as ações da administração pública, quanto aos investimentos realizados, têm efeito positivo nos indicadores de desenvolvimento, considerando a unidade federativa analisada. 
Portanto, esses resultados, além de oferecer informações quanto à atuação dos responsáveis pela gestão dos gastos públicos dos municípios, também apontam possibilidades de investigações para novos estudos como: (i) identificar outras variáveis para analisar o efeito dos gastos públicos nos indicadores de desenvolvimento nos municípios atingidos, quanto às áreas de saúde, infraestrutura, trabalho, segurança e saneamento; (ii) utilizar outra variável dependente, a exemplo do PIB e (iii) considerar a influência do contexto no território de realização do estudo.

\section{AGRADECIMENTOS}

O presente trabalho foi realizado com apoio da Coordenação de Aperfeiçoamento de Pessoal de Nível Superior - Brasil (CAPES) - Código de Financiamento 001. Agradecimentos adicionais ao Grupo de Pesquisa em Gestão e Desenvolvimento de Territórios Criativos (GDTeC) do Núcleo de Administração e Políticas Públicas (NAP2) registrado no Conselho Nacional de Desenvolvimento Científico e Tecnológico (CNPq).

\section{NOTAS}

1- Neste texto utilizaremos autores que se referem ao conceito de desenvolvimento local. Tal concepção, em geral, ressalta os fatores econômicos como indicadores de desenvolvimento. Já outros autores, por exemplo, Dallabrida (2017), Saquet (2015), Pecqueur (2009) e Schneider (2004), utilizam o conceito desenvolvimento territorial, com o propósito de expressar uma compreensão de desenvolvimento em que os fatores econômicos estão na mesma relação de importância de outros, tais como, os fatores histórico-culturais, sociais e ambientais.

2- Compostos pelos seguintes municípios: Araújos, Belo Horizonte, Betim, Bom Despacho, Carmo do Cajuru, Cláudio, Conceição do Pará, Contagem, Divinópolis, Esmeraldas, Guidoval, Igaratinga, Itabira, Itajubá, Itaúna, Jaíba, Janaúba, Lagoa Santa, Leandro Ferreira, Matias Cardoso, Montes Claros, Nova Lima, Nova Porteirinha, Nova Serrana, Pará de Minas, Pedras de Maria da Cruz, Perdigão, Pirapora, Piraúba, Pitangui, Porteirinha, Pouso Alegre, Ribeirão das Neves, Rio Pomba, Rodeiro, Sabará, Santa Luzia, Santa Rita do Sapucaí, São Geraldo, São Gonçalo do Pará, São José da Lapa, Sete Lagoas, Tocantins, Ubá, Varginha, Vespasiano e Visconde do Rio Branco. 


\section{REFERÊNCIAS}

ALBUQUERQUE, Francisco. Desenvolvimento econômico local e distribuição do progresso técnico: uma resposta às exigências do ajuste estrutural. Traduzido por Antônio Rubens Pompeu Braga. Fortaleza: BNB, 1998, 151p.

ALMEIDA, Rodrigo Ferraz de; ALBINO, Andréia Aparecida. Percepção dos empresários sobre governança e desenvolvimento dos APLs de móveis e de confecções de Ubá-MG e região. In: Encontro de Estudos em Estratégia, 6, 2011, Porto Alegre, RS. Anais... Porto Alegre, RS, 2011. p. 1-17.

ANDRADE, Sandro Ângelo de; TEIXEIRA, Arilton; FORTUNATO, Graziela. Influência dos gastos públicos sociais sobre o PIB dos municípios do estado de Minas Gerais. Revista Economia \& Gestão, Belo Horizonte, MG: PUCMINAS, 14, n. 35, p. 112-130, abr./jun. 2014. DOI: https://doi.org/10.5752/P.1984-6606.2014v14n35p112

ANTERO, C. A. S.; RODRIGUES, C. T.; EMMENDOERFER, M. L.; DALLABRIDA, V. R. Política Pública de Apoio ao Desenvolvimento de APLs uma Análise do Impacto em Minas Gerais, Brasil. Cadernos EBAPE. BR, v. 18, n. 1, p. 61-73, 2020. DOI: https://doi.org/10.1590/1679-395176924

AQUINO, André Luiz de; BRESCIANI, Luís Paulo. Arranjos produtivos locais: uma abordagem conceitual. Organizações em Contexto. São Bernardo do Campo, SP, v. 1, n. 2, dez. 2005. DOI: https://doi.org/10.15603/1982-8756/roc.v1n2p\%20153\%20-\%20167

AZEVEDO FILHO, Edson Terra; RIBEIRO, Alcimar Das Chagas. A governança em aglomerações produtivas: uma análise sobre o setor cerâmico de Campos dos Goytacazes. Revista Brasileira de Gestão e Desenvolvimento Regional, Taubaté, SP: Universidade de Taubaté, v. 7, n. 1, p. 96-129, jan./abr. 2011.

BAPTISTA, Josil do Rocio Voidela; ALVAREZ, Victor Manoel Pelaez. Relações socioeconômicas em rede: a governança no Arranjo Produtivo do Vestuário de Cianorte no Estado do Paraná. Revista Paranaense de Desenvolvimento, Curitiba, PR: IPARDES, n.113, p.59-81, jul./dez. 2007.

BARROSO, Janayna Arruda; SOARES, Alexandre Araújo Cavalcante. O impacto das políticas públicas no desenvolvimento de arranjos produtivos locais: o caso do APL de ovino caprinocultura em Quixadá, Ceará. Revista da Administração Pública, Rio de Janeiro, RJ: FGV, v. 43, n. 6, p. 1435-1457, nov./dez. 2009. DOI: http://dx.doi.org/10.1590/S0034$\underline{76122009000600010}$

BOGONI, Nádia Mar; HEIN, Nelson; BEUREN Ilse Maria. Análise da relação entre crescimento econômico e gastos públicos nas maiores cidades da região Sul do Brasil. Revista da Administração Pública, Rio de Janeiro, RJ: FGV, v. 45, n. 1, p. 159-179, jan./fev. 2011. DOI: http://dx.doi.org/10.1590/S0034-76122011000100008

BOTELHO, Marisa dos Reis Azevedo. Análise do mapeamento e das políticas para arranjos produtivos locais no Estado de Minas Gerais. Uberlândia, MG: 2009. (Relatório). 
BRASIL. Ministério do Desenvolvimento, Indústria e Comércio Exterior. Portaria Interministerial $\mathbf{n}^{\circ}$ 200, de 02 de agosto de 2004. Institui o Grupo de Trabalho Permanente para Arranjos Produtivos Locais - GTP APL. Disponível em: http://portalapl.ibict.br/biblioteca/Portaria_Interministerial_numero_200_2004_MDIC.html.

Acesso em: 20 out. 2017.

CAMPOS, Antônio Carlos de; TRINTIN, Jaime Graciano; VIDIGAL, Vinícius Gonçalves. Estrutura de Governança: o caso do arranjo produtivo local (APL) do setor de confecção de Maringá (PR). Textos de Economia, Florianópolis, SC: UFC, v.12, n.1, p.134-155, jan./jun. 2009. DOI: https://doi.org/10.5007/2175-8085.2009v12n1p134

CASSIOLATO, José Eduardo; LASTRES, Helena Maria Martins. Arranjos e sistemas produtivos locais na indústria brasileira. Revista de Economia Contemporânea, Rio de Janeiro, RJ, v. 5, p. 103-136, 2001.

CASTRO, Marcos de; GONÇALVES, Sandro Aparecido. Contexto institucional de referência e governança de redes: estudo em arranjos produtivos locais do estado do Paraná. Revista da Administração Pública, Rio de Janeiro, RJ: FGV v. 48, n. 5, p. 1281-1304, set./out. 2014. DOI: http://dx.doi.org/10.1590/0034-76121764

COMITÉ Económico y Social de Las Comunidades Europeas. Dictamen sobre el desarrollo local en la política regional comunitaria. Bruxelas: CES, 1995.

COSTA, Frederico Lustosa da; CUNHA, Augusto Paulo Guimarães. Pensar o desenvolvimento a partir do local: novo desafio para os gestores públicos. In: Congreso Internacional del CLAD sobre la Reforma del Estado y de la Administración Pública, 7, Lisboa, Portugal. Anais [...] Lisboa, Portugal, 2002, p. 1-13.

DALLABRIDA, Valdir Roque. A abordagem territorial do desenvolvimento: debates, desafios, incongruências e uma proposta. In: DALLABRIDA, Valdir Roque. Teorias do Desenvolvimento: aproximações teóricas que tentam explicar as possibilidades e desafios quanto ao desenvolvimento de lugares, regiões, territórios ou países. Curitiba: Editora CRV, 2017, p. 133-161.

DEGENHART, Larissa; VOGT, Mara; ZONATTO, Vinícius Costa da Silva. Influência dos gastos públicos no crescimento econômico dos municípios da Região Sudeste do Brasil. Revista de Gestão, São Paulo, SP: USP, v. 23, n.3, p. 233-245, mai./jun. 2016. DOI: https://doi.org/10.1016/j.rege.2016.06.005

DIAS, Robson. Institutions and territorial development: a case study from the productive arrangement of oil and natural gas located in Macaé, Brazil. Revista Latinoamericana de Estudios Urbano Regionales - EURE. Santiago de Chile, v. 39, n. 116, p. 141-171. 2013. DOI: http://dx.doi.org/10.4067/S0250-71612013000100006

FUINI, Lucas Labigalini. A governança em arranjos produtivos locais (APLs): algumas considerações teóricas e metodológicas. Geografia. Londrina, PR, v. 23, n.1, p. 57 - 83, jan./jun. 2014. DOI: http://dx.doi.org/10.5433/2447-1747.2014v23n1p57

GARONE, L. F.; MAFFIOLI, A.; NEGRI, J. A.; RODRIGUEZ, C. M.; VÁZQUEZ-BARÉ, G. Cluster development policy, SME's performance, and spillovers: evidence from Brazil. 
Small Business Economics, v. 44, n. $\quad 4, \quad$ p. $925-948, \quad 2015 . \quad$ DOI: https://doi.org/10.1007/s11187-014-9620-2

HASENCLEVER, Lia; ZISSIMOS, Isleide. A evolução das configurações produtivas locais no Brasil: uma revisão da literatura. Estudos Econômicos, São Paulo, SP, v. 36, n. 3, p. 407433, 2006.

HILL, R. Carter; GRIFFITHS, William E.; LIM, Guay C. Principles of econometrics. 4. ed. Hoboken, NJ: Wiley, 2011.

JACOMETTI, Márcio; CASTRO, Marcos de; GONÇALVES, Sandro Aparecido; COSTA, Mayla Cristina. Análise de efetividade das políticas públicas de Arranjo Produtivo Local para o desenvolvimento local a partir da teoria institucional. Revista da Administração Pública, Rio de Janeiro, RJ: FGV, v. 50, n. 3, p. 425-454, mai./jun. 2016. DOI: https://doi.org/10.1590/0034-7612142712

KACHBA, Yslene Rocha; HATAKEYAMA, Kazuo. Estratégias de inovação em APLs: viés para o desenvolvimento de produtos de moda. Produção, São Paulo, SP, v. 23, n. 4, p. 751761, out./dez. 2013. DOI: https://doi.org/10.1590/S0103-65132013005000012

KREUZ, Carlos Leomar; SOUZA, Alceu; CUNHA, Sieglind Kindl da; PERFEITO, Juarez. Indicadores de viabilidade e estratégias competitivas: o caso dos produtores de alho na região de Curitibanos-SC. Revista Alcance. Palmas, PR, v. 12, n. 2, p. 269 - 283, mai./ago. 2005. DOI: https://doi.org/10.14210/alcance.v12n2.p269-284

KÜHN, Daniela Dias; TONETTO, Elci da Silva. O Programa Bolsa Família e os Indicadores Sociais de Combate à Pobreza no Rio Grande do Sul: Um Olhar Multidimensional. Desenvolvimento em Questão, Ijuí, RS: UNIJUI, v. 15, n. 39, p. 86-111, 2017. DOI: https://doi.org/10.21527/2237-6453.2017.39.86-111

LASTRES, Helena Maria Martins; CASSIOLATO, José Eduardo. Glossário de arranjos e sistemas produtivos e inovativos locais. Nov. 2003. Disponível em: www.redesist.ie.ufrj.br. Acesso em: 24 mai. 2013.

MACEDO, Roberto Aparecido; ARAÚJO, Luiz Jurandir Simões de; CAMPOS, Anderson Luís Saber. Clusters de pequenas e médias empresas e desenvolvimento regional. Revista Eletrônica Gestão e Serviços, São Bernardo do Campo, SP: Universidade Metodista de São Paulo, v. 5, n. 2, p. 1-19, jul./dez. 2014. DOI: https://doi.org/10.15603/21777284/regs.v5n2p1014-1032

MARIA, Ana Sílvia Rocha Ipiranga; FARIA, Vilma Coelho Moreira; AMORIM, Mônica Alves. A comunidade de prática da rede nós: colaborando e compartilhando conhecimentos em arranjos produtivos locais. Organização \& Sociedade. Salvador, BA, v.15, n.44, jan./mar. 2008. DOI: https://doi.org/10.1590/S1984-92302008000100008

MARSHALL, Alfred. Princípios de economia. São Paulo: Abril Cultural, 1982. 272 p.

MARTINS, Sérgio Ricardo Oliveira. Desenvolvimento Local: questões conceituais e metodológicas. Interações - Revista Internacional de Desenvolvimento Local, Campo Grande, MS: UCDB v. 3, n. 5, p. 51-59, set. 2002. DOI: https://doi.org/10.20435/interacoes.v3i5.570 
MEDEIROS, Ana Lucia; DOS SANTOS, Luana Borges; ANDRE, Claudomiro Moura Gomes. Desenvolvimento Municipal das Microrregiões do Estado do Tocantins: uma análise a partir do Índice Firjan de Desenvolvimento Municipal. Desenvolvimento em Questão, Ijuí, RS: UNIJUI, v. 16, n. 45, p. 44-62, 2018. DOI: https://doi.org/10.21527/2237$\underline{6453.2018 .45 .44-62}$

MINAS GERAIS. Decreto 44.972, de 02 de dezembro de 2008. Regulamenta as ações da Política Estadual de Apoio aos Arranjos Produtivos Locais e dá outras providências. Belo Horizonte. 2008.

Disponível em: http://www.almg.gov.br/consulte/legislacao/completa/completa.html?tipo=DEC\&num=44972 $\underline{\& c o m p}=\& a n o=2008 \& a b a=j$ s textoOriginal\#texto. Acesso em: 07 ago. 2015.

MINAS GERAIS. Lei 16.296, de 01 de agosto de 2006. Institui a Política Estadual de Apoio aos Arranjos Produtivos Locais e dá Outras Providências. Belo Horizonte. 2006. Disponível em: $\quad$ http://leisestaduais.com.br/mg/lei-ordinaria-n-16296-2006-minas-gerais-institui-apolitica-estadual-de-apoio-aos-arranjos-produtivos-locais-e-da-outras-providencias. Acesso em: 20 out. 2017.

MINAS GERAIS. Secretaria de Desenvolvimento Econômico. Programa de Apoio à Competitividade dos Arranjos Produtivos Locais de Minas Gerais. Belo Horizonte. 2015. Disponível em: http://www.fiemg.org.br/Default.aspx?tabid=11854. Acesso em 10 ago. 2015.

NEDUZIAK, Luiz Carlos Ribeiro; CORREIA, Fernando Motta. Alocação dos Gastos Públicos e Crescimento Econômico: Um Estudo em Painel para os Estados Brasileiros. Revista de Administração Pública, Rio de Janeiro, RJ: FGV, v. 51, n. 4, p. 616-632, 2017. DOI: https://doi.org/10.1590/0034-7612155177

OLIVEIRA, Cyntia Meireles de; SANTANA, Antônio Cordeiro de. A Governança no Arranjo Produtivo de Grãos de Santarém e Belterra, Estado do Pará: uma análise a partir do grão soja. Revista de Economia e Sociologia Rural, Piracicaba, SP: SOBER, v. 50, n. 4, p. 683-704, out./dez. 2012. DOI: http://dx.doi.org/10.1590/S0103-20032012000400006

PASSOS, Francisco Huchoa; DIAS, Camila Carneiro; CRUZ; Rossine Cerqueira da. Capital social, competências e demandas tecnológicas de arranjos produtivos locais: o caso do APL de sisal em Valente, Bahia. Economia \& Gestão. Belo Horizonte, MG, v. 5, n. 10, p. 92-112, ago. 2005.

PECQUEUR, Bernard. A guinada territorial da economia global. Política \& Sociedade, Florianópolis, SC: UFSC, n. 14, p. 79-105, abril, 2009. DOI: https://doi.org/10.5007/2175$\underline{7984.2009 \mathrm{v} 8 \mathrm{n} 14 \mathrm{p} 79}$

PEREIRA, Camila Cristina de Paula; GUIMARÃES, Liliane de Oliveira; GOMES, Miguel Ângelo. Governança de arranjos produtivos locais e suas possibilidades. In: Encontro da ANPAD, 35, 2011, Rio de Janeiro, RJ. Anais [...] Rio de Janeiro, RJ, 2011. p. 1-17.

SANTOS, Aysa Eliude Aguiar dos; CÂNDIDO, Gesinaldo Ataíde. Estruturas de governança em arranjos produtivos locais: uma aplicação no arranjo calçadista no município de Campina Grande-PB. In: Encontro da ANPAD, 37, 2013, Rio de Janeiro, RJ. Anais... Rio de Janeiro, RJ, 2013. p. 1-16. 
SANTOS, Gustavo Antônio Galvão dos; DINIZ, Eduardo José; BARBOSA, Eduardo Kaplan. Aglomerações, arranjos produtivos locais e vantagens competitivas locacionais. Revista do BNDES, Rio de Janeiro, RJ: BNDES, v. 11, n. 22, p. 151-179, dez. 2004.

SAQUET, Marcos. Por uma geografia das territorialidades e das temporalidades. Uma concepção multidimensional voltada para a cooperação e para o desenvolvimento territorial. 2.ed. rev. e ampl. Rio de Janeiro: Ed. Consequência, 2015.

SCHMITZ, Hubert. Eficiência coletiva: caminho de crescimento para a indústria de pequeno porte. Ensaios EFE, Porto Alegre, RS, v. 18, n. 2, p. 164-200, 1997.

SCHNEIDER, Sérgio. A abordagem territorial do desenvolvimento e suas articulações externas. Sociologias, Porto Alegre, RS: UFRGS, v. 6, n. 11, p. 88-125, jan./jun., 2004. DOI: https://doi.org/10.1590/S1517-45222004000100006

SCHULL, Adiulli Natã; FEITÓSA, Camila Gonçalves; HEIN, André Fernando. Análise da eficiência dos gastos em segurança pública nos estados brasileiros através da Análise Envoltória de Dados (DEA). Revista Capital Científico - Eletrônica, Guarapuava, PR: UNICENTRO, v. 12, n. 3, p. 91-105, 2014. DOI: https://doi.org/10.5935/2177$\underline{4153.20140023}$

SEBRAE. Serviço Brasileiro de Apoio à Micro e Pequena Empresa. Termo de referência para atuação do SEBRAE em arranjos produtivos locais. Brasília: SEBRAE, 2003.

SOUSA, Rossana Guerra; PAULO, Edilson. Produtividade dos Gastos Públicos dos Estados Brasileiros: Um Estudo Empírico no Período de 1995 a 2010. Revista Desenvolvimento em Questão, Ijuí, RS: UNIJUI, v. 14, n. 36, p. 187-219, out./dez., 2016. DOI: https://doi.org/10.21527/2237-6453.2016.36.187-219

SOUZA, Paulo Augusto Ramalho de; ROMEIRO, Maria do Carmo; AMADOR, César Augusto; OLIVEIRA Suellen Moreira de; ZAMBRA Elisandra Marisa. The transfer of information for the development of small-scale fishing in the Amazon. Interciencia. Caracas, Venezuela, v. 40, n. 1, p. 44-49. 2015.

SUGAHARA, Cibele Roberta; VERGUEIRO, Waldomiro de Castro Santos. Information and knowledge: an analysis in the context of business network. Revista Interamericana de Bibliotecología. Medellín, Colômbia. v. 35 n. 2, p. 163-171, May/Aug. 2012.

SUZIGAN, Wilson; FURTADO, João, GARCIA, Renato; SAMPAIO, Sergio. Sistemas locais de produção: mapeamento, tipologia e sugestões de políticas. In: Encontro Nacional de Economia, 33, 2003, Porto Seguro, BA. Anais... Porto Seguro, BA, 2003, p. 1-29.

TAHIM, Elda Fontinele; ARAÚJO JUNIOR, Inácio Fernandes de. A carcinicultura do Nordeste Brasileiro e sua Inserção em Cadeias Globais de Produção: foco nos APLs do Ceará. Revista e Economia e Sociologia Rural. Piracicaba, SP, v. 52, n. 3, p. 567-586, jul./set. 2014. DOI: https://doi.org/10.1590/S0103-20032014000300009

TEIXEIRA, Francisco. Políticas públicas para o desenvolvimento regional e local: o que podemos aprender com os arranjos produtivos locais (APLS)? Organizações \& Sociedade, Salvador, BA: UFBA, v. $15, \quad$ n. $46, \quad$ p. 57 - 75, jun./set. 2008. DOI: https://doi.org/10.1590/S1984-92302008000300003 
VALE, Gláucia Maria Vasconcellos. Aglomerações produtivas: tipologias de análises e repercussões nos estudos organizacionais. Organização \& Sociedade. Salvador, BA, v.14, n.43, p.159-175, out./dez. 2007. DOI: https://doi.org/10.1590/S1984-92302007000400009

ZAMBRANA, Aline de Aragão; TEIXEIRA, Rivanda Meira. Governança e cooperação em arranjos produtivos locais: um estudo de múltiplos casos em Sergipe. REGE-Revista de Gestão, v. 20, n. 1, p. 21-41, 2013. DOI: https://doi.org/10.5700/rege485 